Turk. J. Math. Comput. Sci.

13(2)(2021) 359-372

(C) MatDer

DOI : $10.47000 /$ tjmcs. 954676

\title{
A New Type Multivariable Multiple Hypergeometric Functions
}

\author{
Duriye Korkmaz DuzGun ${ }^{1}$ iD \\ ${ }^{1}$ Department of Business Administration(Quantative Methods), Faculty of Economics and Administrative Sciences, Kafkas \\ University, 36000,Kars, Turkey.
}

Received: 19-06-2021 • Accepted: 10-12-2021

Aвstract. We define a new type of multivariable multiple hypergeometric functions in this paper, which is inspired by Exton's multiple hypergeometric functions given by in [13]. Then, for these functions, we obtain some certain type linear generating functions. After that, we derive a variety classes of multilinear and multilateral generating functions for a family of the multivariable multiple hypergeometric functions. In addition, by employing the Erkus-Srivastava polynomials (see [11]) and the fourth type multivariable Horn functions (see [13]), we have also provided some of its conclusions.

2010 AMS Classification: $33 \mathrm{C} 65,33 \mathrm{C} 05,33 \mathrm{C} 20$

Keywords: Multiple hypergeometric functions, multivariable Horn functions, Lauricella functions, Appell functions, generating functions, multilinear and multilateral generating functions, Pochhammer symbol.

\section{INTRODUCTION}

The generalized hypergeometric series ${ }_{p} F_{q}$ with an arbitrary number of $p$ numerator and $q$ denominator parameters $\left(p, q \in N_{0}=\{0\} \cup \mathbb{N}\right)$ defined by [29]

$$
{ }_{p} F_{q}\left[\begin{array}{c}
\alpha_{1}, \ldots, \alpha_{p} ; \\
\beta_{1}, \ldots, \beta_{q} ;
\end{array}\right]={ }_{p} F_{q}\left(\alpha_{1}, \ldots, \alpha_{p} ; \beta_{1}, \ldots, \beta_{q} ; z\right)=\sum_{n=0}^{\infty} \frac{\left(\alpha_{1}\right)_{n} \ldots\left(\alpha_{p}\right)_{n}}{\left(\beta_{1}\right)_{n} \ldots\left(\beta_{q}\right)_{n}} \frac{z^{n}}{n !},
$$

where the Pochhammer symbol is denoted by $(\lambda)_{v}$ which is defined (in terms of gamma function) by

$$
\begin{aligned}
(\lambda)_{v} & =\frac{\Gamma(\lambda+v)}{\Gamma(\lambda)}\left(\lambda \in \mathbb{C} \backslash \mathbb{Z}_{0}^{-}\right) \\
& = \begin{cases}1, & \text { if } v=0 ; \lambda \in \mathbb{C} \backslash\{0\} \\
\lambda(\lambda+1) \ldots(\lambda+n-1), & \text { if } v=n \in \mathbb{N} ; \lambda \in \mathbb{C},\end{cases}
\end{aligned}
$$

$\Gamma(\lambda)$ is Gamma function, and $Z_{0}^{-}$denotes the set of non-positive integers. If we set $p=2, q=1$ in (1.1), we get a hypergeometric function following,

$$
{ }_{2} F_{1}(\alpha, \beta ; \gamma ; z)=\sum_{n=0}^{\infty} \frac{(\alpha)_{n}(\beta)_{n}}{(\gamma)_{n}} \frac{z^{n}}{n !}
$$

Email address: dryekorkmaz@gmail.com; dkduzgun@kafkas.edu.tr (D. Korkmaz Düzgün) 
and if we also set $p=1, q=1$, we get a confluent hypergeometric function as follow,

$$
\Phi={ }_{1} F_{1}(\beta ; \gamma ; z)=\sum_{n=0}^{\infty} \frac{(\beta)_{n}}{(\gamma)_{n}} \frac{z^{n}}{n !} .
$$

In addition, in (1.1), the absence of parameters $p$ or $q$ is emphasized by a dash (interpreting an empty product as 1 ). For example, if no numerator or denominator parameters are present, i.e., $p=q=0$, the result is

$$
{ }_{0} F_{0}(-;-; z)=\sum_{n=0}^{\infty} \frac{z^{n}}{n !} \text {. }
$$

Similarly if $p=0$ and $q=1$, then

$$
{ }_{0} F_{1}(-; \beta ; z)=\sum_{n=0}^{\infty} \frac{z^{n}}{(\beta)_{n} n !}
$$

and finally if $p=1$ and $q=0$, then

$$
{ }_{1} F_{0}(\alpha ;-; z)=\sum_{n=0}^{\infty} \frac{(\alpha)_{n} z^{n}}{n !}
$$

The second type of Appell functions, the first type of Lauricella functions, the fourth type of Horn functions, and the fourth type of multivariable Horn functions are, respectively [13, 16, 21, 29],

$$
F_{2}\left(\alpha, \beta_{1}, \beta_{2} ; \gamma_{1}, \gamma_{2} ; x_{1}, x_{2}\right)=\sum_{m, r=0}^{\infty}(\alpha)_{m+r} \frac{\left(\beta_{1}\right)_{m}\left(\beta_{2}\right)_{r}}{\left(\gamma_{1}\right)_{m}\left(\gamma_{2}\right)_{r}} \frac{x_{1}^{m}}{m !} \frac{x_{2}^{r}}{r !}
$$

where $\quad\left|x_{1}\right|+\left|x_{2}\right|<1$, and

$$
F_{A}^{(r)}\left(\alpha, \beta_{1}, \ldots, \beta_{r} ; \gamma_{1}, \ldots, \gamma_{r} ; x_{1}, \ldots, x_{r}\right)=\sum_{m_{1}, \ldots, m_{r}=0}^{\infty}(\alpha)_{m_{1}+\ldots+m_{r}} \frac{\left(\beta_{1}\right)_{m_{1}} \ldots\left(\beta_{r}\right)_{m_{r}}}{\left(\gamma_{1}\right)_{m_{1}} \ldots\left(\gamma_{r}\right)_{m_{r}}} \frac{x_{1}^{m_{1}}}{m_{1} !} \ldots \frac{x_{r}^{m_{r}}}{m_{r} !},
$$

where $\left|x_{1}\right|+\ldots+\left|x_{r}\right|<1$, and

$$
H_{4}\left(\alpha, \beta ; \gamma_{1}, \gamma_{2} ; x_{1}, x_{2}\right)=\sum_{m, r=0}^{\infty} \frac{(\alpha)_{2 m+r}(\beta)_{r}}{\left(\gamma_{1}\right)_{m}\left(\gamma_{2}\right)_{r}} \frac{x_{1}^{m}}{m !} \frac{x_{2}^{r}}{r !}
$$

where $2 \sqrt{\left|x_{1}\right|}+\left|x_{2}\right|<1$, and

$$
\begin{aligned}
{ }^{(k)} H_{4}^{(r)} & \left(\alpha, \beta_{k+1}, \ldots, \beta_{r} ; \gamma_{1}, \ldots, \gamma_{r} ; x_{1}, \ldots, x_{k}, x_{k+1}, \ldots, x_{r}\right) \\
& =\sum_{m_{1}, \ldots, m_{r}=0}^{\infty} \frac{(\alpha)_{2\left(m_{1}+\ldots+m_{k}\right)+m_{k+1}+\ldots+m_{r}}\left(\beta_{k+1}\right)_{m_{k+1}} \ldots\left(\beta_{r}\right)_{m_{r}}}{\left(\gamma_{1}\right)_{m_{1}} \ldots\left(\gamma_{r}\right)_{m_{r}}} \frac{x_{1}^{m_{1}}}{m_{1} !} \ldots \frac{x_{r}^{m_{r}}}{m_{r} !},
\end{aligned}
$$

where $2\left(\sqrt{\left|x_{1}\right|}+\ldots+\sqrt{\left|x_{k}\right|}\right)+\left|x_{k+1}\right|+\ldots+\left|x_{r}\right|<1$.

The multivariable multiple hypergeometric functions are highly significant in special functions theory, used in a wide range of applications, such as integral representations, generating functions, recurrence relations, finite and infinite sums, analytic continuation, asymptotic behaviour and some special formulas (linear transformation, quadratic transformation, decomposition, reduction, limit and differentiation) [1,4-7,10,13,16,21]. Additionally, various types of extensions (q-analogue, k-analogue, finite field analogue, matrix extension, p-extension) of these functions are defined, and similar features are extensively researched $[2,3,9,12,14,15,18-20,23,30]$. Besides from these studies, they are also utilized in theories such as perturbation theory (in numerical analysis) and quantum theory (in modern physics) [17,24]. Studies of generating functions for polynomial sequences, on the other hand, are widely investigated, which typically comprise ordinary, multilinear and multilateral generating functions $[8,11,22,25,26,28]$.

In this paper, we described a new type of multivariable multiple hypergeometric functions that are general forms of the fourth type multivariable Horn functions ${ }^{(k)} H_{4}^{(r)}$, the first kind Lauricella $F_{A}^{(r)}$, the second kind Appell $F_{2}$ and the fourth type Horn $\mathrm{H}_{4}$ functions. A similar idea was followed by Exton in [13] to study the generating functions of the fourth type multivariable Horn functions. Our objective is to derive obtaining certain linear generating functions and several families of multilinear and multilateral generating functions, as well as their conclusions, for these multivariable multiple hypergeometric functions. 


\section{Generating Functions}

We obtain certain linear generating function relations after defining a new type of multivariable multiple hypergeometric functions in this section of the study. Below is a definition of a new type of multivariable multiple hypergeometric functions.

Definition 2.1. We define a new type multivariable multiple hypergeometric function as below:

$$
{ }^{(k)} E^{(r)}\left(\alpha, \beta_{k+1}, \ldots, \beta_{r} ; \gamma_{1}, \ldots, \gamma_{r} ; x_{1}, \ldots, x_{r}\right):=\sum_{m_{1}, \ldots, m_{r}=0}^{\infty}(\alpha)_{\rho\left(m_{1}+\ldots+m_{k}\right)+m_{k+1}+\ldots+m_{r}} \frac{\left(\beta_{k+1}\right)_{m_{k+1}} \ldots\left(\beta_{r}\right)_{m_{r}}}{\left(\gamma_{1}\right)_{m_{1}} \ldots\left(\gamma_{r}\right)_{m_{r}}} \frac{x_{1}^{m_{1}}}{m_{1} !} \ldots \frac{x_{r}^{m_{r}}}{m_{r} !},
$$

where $\rho\left(\sqrt{\left|x_{1}\right|}+\ldots+\sqrt{\left|x_{k}\right|}\right)+\left|x_{k+1}\right|+\ldots+\left|x_{r}\right|<1, \rho \geqslant 0$.

In (2.1), if we take $k=1, r=2$, we have two-variable multiple hypergeometric functions as follow

$$
{ }^{(1)} E^{(2)}\left(\alpha, \beta_{2} ; \gamma_{1}, \gamma_{2} ; x_{1}, x_{2}\right)=E\left(\alpha, \beta_{2} ; \gamma_{1}, \gamma_{2} ; x_{1}, x_{2}\right):=\sum_{m_{1}, m_{2}=0}^{\infty}(\alpha)_{\rho m_{1}+m_{2}} \frac{\left(\beta_{2}\right)_{m_{2}}}{\left(\gamma_{1}\right)_{m_{1}}\left(\gamma_{2}\right)_{m_{2}}} \frac{x_{1}^{m_{1}}}{m_{1} !} \frac{x_{2}^{m_{2}}}{m_{2} !} .
$$

For the functions given (2.1), some generating function relations have been established in various ways, as well as some results.

Theorem 2.2. We have the following generating function for the multivariable multiple hypergeometric function ${ }^{(k)} E^{(r)}$ defined by (2.1):

$$
\begin{aligned}
\sum_{n=0}^{\infty} \frac{(\lambda)_{n}}{n !}{ }^{(k)} E^{(r)}\left(\lambda+n, \beta_{k+1}, \ldots, \beta_{r} ; \gamma_{1}, \ldots, \gamma_{r} ; x_{1}, \ldots, x_{r}\right) t^{n}=(1-t)^{-\lambda} \\
\quad \times{ }^{(k)} E^{(r)}\left(\lambda, \beta_{k+1}, \ldots, \beta_{r} ; \gamma_{1}, \ldots, \gamma_{r} ; \frac{x_{1}}{(1-t)^{\rho}}, \ldots, \frac{x_{k}}{(1-t)^{\rho}}, \frac{x_{k+1}}{(1-t)}, \ldots, \frac{x_{r}}{(1-t)}\right),
\end{aligned}
$$

where $\lambda \in \mathbb{C},|t|<1$.

Proof. Let $T$ denote the first member of assertion (2.3),

$$
\begin{aligned}
T & =\sum_{n=0}^{\infty} \frac{(\lambda)_{n}{ }^{(k)} E^{(r)}\left(\lambda+n, \beta_{k+1}, \ldots, \beta_{r} ; \gamma_{1}, \ldots, \gamma_{r} ; x_{1}, \ldots, x_{r}\right) t^{n}}{n !} \\
& =\sum_{n=0}^{\infty} \sum_{m_{1}, \ldots, m_{r}=0}^{\infty}(\lambda)_{n}(\lambda+n)_{\rho\left(m_{1}+\ldots+m_{k}\right)+\ldots+m_{k+1}+\ldots+m_{r}} \frac{\left(\beta_{k+1}\right)_{m_{k+1}} \ldots\left(\beta_{r}\right)_{m_{r}}}{\left(\gamma_{1}\right)_{m_{1}} \ldots\left(\gamma_{r}\right)_{m_{r}}} \frac{\left(x_{1}\right)^{m_{1}}}{m_{1} !} \ldots \frac{\left(x_{r}\right)^{m_{r}}}{m_{r} !} \frac{t^{n}}{n !} .
\end{aligned}
$$

Using the identity [27, page 87]

$$
(\lambda)_{n}(\lambda+n)_{k}=(\lambda)_{n+k}=(\lambda)_{k}(\lambda+k)_{n},
$$

with $k=\rho\left(m_{1}+\ldots+m_{k}\right)+\ldots+m_{k+1}+\ldots+m_{r}$ in $(2.5)$, we have

$$
\begin{aligned}
(\lambda)_{n} & (\lambda+n)_{\rho\left(m_{1}+\ldots+m_{k}\right)+\ldots+m_{k+1}+\ldots+m_{r}} \\
& =(\lambda)_{n+\rho\left(m_{1}+\ldots+m_{k}\right)+\ldots+m_{k+1}+\ldots+m_{r}} \\
& =(\lambda)_{\rho\left(m_{1}+\ldots+m_{k}\right)+\ldots+m_{k+1}+\ldots+m_{r}}\left(\lambda+\rho\left(m_{1}+\ldots+m_{k}\right)+m_{k+1}+\ldots+m_{r}\right)_{n} .
\end{aligned}
$$

Thus, we can write (2.4)

$$
\begin{aligned}
T=\sum_{m_{1}, \ldots, m_{r}=0}^{\infty}(\lambda)_{\rho\left(m_{1}+\ldots+m_{k}\right)+\ldots+m_{k+1}+\ldots+m_{r}} \frac{\left(\beta_{k+1}\right)_{m_{k+1}} \ldots\left(\beta_{r}\right)_{m_{r}}}{\left(\gamma_{1}\right)_{m_{1}} \ldots\left(\gamma_{r}\right)_{m_{r}}} \\
\quad \times \frac{\left(x_{1}\right)^{m_{1}}}{m_{1} !} \ldots \frac{\left(x_{r}\right)^{m_{r}}}{m_{r} !} \sum_{n=0}^{\infty} \frac{\left(\lambda+\rho\left(m_{1}+\ldots+m_{k}\right)+m_{k+1}+\ldots+m_{r}\right)_{n}}{n !} t^{n} .
\end{aligned}
$$

Using the identity [27, page 58]

$$
(1-t)^{-c}=\sum_{n=0}^{\infty} \frac{(c)_{n}}{n !} t^{n},
$$


with $c=\lambda+\rho\left(m_{1}+\ldots+m_{k}\right)+m_{k+1}+\ldots+m_{r}$ in $(2.7)$, we obtain

$$
\sum_{n=0}^{\infty} \frac{\left(\lambda+\rho\left(m_{1}+\ldots+m_{k}\right)+m_{k+1}+\ldots+m_{r}\right)_{n}}{n !} t^{n}=(1-t)^{-\lambda-\rho\left(m_{1}+\ldots+m_{k}\right)-m_{k+1}-\ldots-m_{r}}
$$

Thus, we can write (2.6) as follows,

$$
\begin{aligned}
T=(1-t)^{-\lambda} & \sum_{m_{1}, \ldots, m_{r}=0}^{\infty}(\lambda)_{\rho\left(m_{1}+\ldots+m_{k}\right)+\ldots+m_{k+1}+\ldots+m_{r}} \frac{\left(\beta_{k+1}\right)_{m_{k+1}} \ldots\left(\beta_{r}\right)_{m_{r}}}{\left(\gamma_{1}\right)_{m_{1}} \ldots\left(\gamma_{r}\right)_{m_{r}}} \\
& \times\left[\frac{x_{1}}{(1-t)^{\rho}}\right]^{m_{1}} \ldots\left[\frac{x_{k}}{(1-t)^{\rho}}\right]^{m_{k}}\left[\frac{x_{k+1}}{(1-t)}\right]^{m_{k+1}} \ldots\left[\frac{x_{r}}{(1-t)}\right]^{m_{r}} \frac{1}{m_{1} !} \ldots \frac{1}{m_{r} !} .
\end{aligned}
$$

If we apply the definition (2.1), we get the desired equality, and the proof is complete.

If we take $k=1, r=2$ in Theorem 2.2), we have the following conclusion for the two-variable multiple hypergeometric functions given by Equation (2.2).

Corollary 2.3. If we take $k=1, r=2$ in Theorem 2.2, then the two-variable multiple hypergeometric functions in Equation 2.2 have the following relation:

$$
\sum_{n=0}^{\infty} \frac{(\lambda)_{n}}{n !} E\left(\lambda+n, \beta_{2} ; \gamma_{1}, \gamma_{2} ; x_{1}, x_{2}\right) t^{n}=(1-t)^{-\lambda} E\left(\lambda, \beta_{2} ; \gamma_{1}, \gamma_{2} ; \frac{x_{1}}{(1-t)^{\rho}}, \frac{x_{2}}{(1-t)}\right)
$$

where $\lambda \in \mathbb{C}$ and $|t|<1$.

If we set $\rho=2$ in (2.1), we clearly see that the multivariable multiple hypergeometric functions are a generalization of the fourth kind multivariable Horn functions ${ }^{(k)} H_{4}^{(r)}[13]$ :

$$
{ }^{(k)} E^{(r)}\left(\alpha, \beta_{k+1}, \ldots, \beta_{r} ; \gamma_{1}, \ldots, \gamma_{r} ; x_{1}, \ldots, x_{r}\right)={ }^{(k)} H_{4}^{(r)}\left(\alpha, \beta_{k+1}, \ldots, \beta_{r} ; \gamma_{1}, \ldots, \gamma_{r} ; x_{1}, \ldots, x_{r}\right)
$$

Corollary 2.4. If we take $\rho=2$ in Theorem 2.2, then we have the following relation for the fourth kind multivariable Horn functions [13]:

$$
\begin{aligned}
\sum_{n=0}^{\infty} \frac{(\lambda)_{n}}{n !} & { }^{(k)} H_{4}^{(r)}\left(\lambda+n, \beta_{k+1}, \ldots, \beta_{r} ; \gamma_{1}, \ldots, \gamma_{r} ; x_{1}, \ldots, x_{r}\right) t^{n}=(1-t)^{-\lambda} \\
& \times{ }^{(k)} H_{4}^{(r)}\left(\lambda, \beta_{k+1}, \ldots, \beta_{r} ; \gamma_{1}, \ldots, \gamma_{r} ; \frac{x_{1}}{(1-t)^{2}}, \ldots, \frac{x_{k}}{(1-t)^{2}}, \frac{x_{k+1}}{(1-t)}, \ldots, \frac{x_{r}}{(1-t)}\right)
\end{aligned}
$$

where $\lambda \in \mathbb{C}$ and $|t|<1$.

If we choose $k=1, r=2$ in Corollary 2.4, we immediately have the following conclusion for the fourth kind Horn functions [16,29].

Remark 2.5. We have

$$
\sum_{n=0}^{\infty} \frac{(\lambda)_{n}}{n !} H_{4}\left(\lambda+n, \beta ; \gamma_{1}, \gamma_{2} ; x_{1}, x_{2}\right) t^{n}=(1-t)^{-\lambda} H_{4}\left(\lambda, \beta ; \gamma_{1}, \gamma_{2} ; \frac{x_{1}}{(1-t)^{2}}, \frac{x_{2}}{(1-t)}\right)
$$

where $\lambda \in \mathbb{C}$ and $|t|<1$.

If we set $k=0$ in Corollary 2.4, we have the following relation for the first kind Lauricella functions in [21,29].

Remark 2.6. We have

$$
\sum_{n=0}^{\infty} \frac{(\lambda)_{n}}{n !} F_{A}^{(r)}\left(\lambda+n, \beta_{1}, \ldots, \beta_{r} ; \gamma_{1}, \ldots, \gamma_{r} ; x_{1}, \ldots, x_{r}\right) t^{n}=(1-t)^{-\lambda} F_{A}^{(r)}\left(\lambda, \beta_{1}, \ldots, \beta_{r} ; \gamma_{1}, \ldots, \gamma_{r} ; \frac{x_{1}}{(1-t)}, \ldots, \frac{x_{r}}{(1-t)}\right)
$$

where $\lambda \in \mathbb{C}$ and $|t|<1$.

If we choose $k=0, r=2$ in Corollary 2.4, we immediately have the following conclusion for the second kind Appell functions [21,29]. 
Remark 2.7. We have the following generating function for the second kind Appell functions:

$$
\sum_{n=0}^{\infty} \frac{(\lambda)_{n}}{n !} F_{2}\left(\lambda+n, \beta_{1}, \beta_{2} ; \gamma_{1}, \gamma_{2} ; x_{1}, x_{2}\right) t^{n}=(1-t)^{-\lambda} F_{2}\left(\lambda, \beta_{1}, \beta_{2} ; \gamma_{1}, \gamma_{2} ; \frac{x_{1}}{(1-t)}, \frac{x_{2}}{(1-t)}\right) \text {, }
$$

where $\lambda \in \mathbb{C}$ and $|t|<1$.

Theorem 2.8. We have the following generating function for the multivariable multiple hypergeometric functions ${ }^{(k)} E^{(r)}$ defined by (2.1):

$$
\begin{aligned}
\sum_{n=0}^{\infty} \frac{(\lambda)_{n}}{n !}{ }^{(k)} E^{(r)}( & \left.-n, \beta_{k+1}, \ldots, \beta_{r} ; \gamma_{1}, \ldots, \gamma_{r} ; x_{1}, \ldots, x_{r}\right) t^{n}=(1-t)^{-\lambda} \\
& \times{ }^{(k)} E^{(r)}\left(\lambda, \beta_{k+1}, \ldots, \beta_{r} ; \gamma_{1}, \ldots, \gamma_{r} ; \frac{x_{1}(-t)^{\rho}}{(1-t)^{\rho}}, \ldots, \frac{x_{k}(-t)^{\rho}}{(1-t)^{\rho}}, \frac{-x_{k+1} t}{(1-t)}, \ldots, \frac{-x_{r} t}{(1-t)}\right),
\end{aligned}
$$

where $\lambda \in \mathbb{C},|t|<1$.

Proof. Let $S$ denote the first member of assertion (2.9). Then,

$$
\begin{aligned}
S & =\sum_{n=0}^{\infty} \frac{(\lambda)_{n}}{n !}{ }^{(k)} E^{(r)}\left(-n, \beta_{k+1}, \ldots, \beta_{r} ; \gamma_{1}, \ldots, \gamma_{r} ; x_{1}, \ldots, x_{r}\right) t^{n} \\
& =\sum_{n=0}^{\infty} \sum_{m_{1}, \ldots, m_{r}=0}^{\rho\left(m_{1}+\ldots+m_{k}\right)+m_{k+1}+\ldots+m_{r} \leq n}(\lambda)_{n}(-n)_{\rho\left(m_{1}+\ldots+m_{k}\right)+m_{k+1}+\ldots+m_{r}} \frac{\left(\beta_{k+1}\right)_{m_{k+1}} \ldots\left(\beta_{r}\right)_{m_{r}}}{\left(\gamma_{1}\right)_{m_{1}} \ldots\left(\gamma_{r}\right)_{m_{r}}} \frac{\left(x_{1}\right)^{m_{1}}}{m_{1} !} \ldots \frac{\left(x_{r}\right)^{m_{r}}}{m_{r} !} \frac{t^{n}}{n !} .
\end{aligned}
$$

Using the idendity [27, page 58]

$$
\frac{(-n)_{k}}{n !}=\frac{(-1)^{k}}{(n-k) !}
$$

with $k=\rho\left(m_{1}+\ldots+m_{k}\right)+m_{k+1}+\ldots+m_{r}$ in $(2.11)$, we get

$$
\frac{(-n)_{\rho\left(m_{1}+\ldots+m_{k}\right)+m_{k+1}+\ldots+m_{r}}}{n !}=\frac{(-1)^{\rho\left(m_{1}+\ldots+m_{k}\right)+m_{k+1}+\ldots+m_{r}}}{\left(n-\rho\left(m_{1}+\ldots+m_{k}\right)-m_{k+1}-\ldots-m_{r}\right) !} .
$$

Thus we can rewrite (2.10)

$$
\begin{aligned}
S=\sum_{n=0}^{\infty} \sum_{m_{1}, \ldots, m_{r}=0}^{\rho\left(m_{1}+\ldots+m_{k}\right)+m_{k+1}+\ldots+m_{r} \leq n} \frac{(\lambda)_{n}\left(\beta_{k+1}\right)_{m_{k+1}} \ldots\left(\beta_{r}\right)_{m_{r}}}{\left(\gamma_{1}\right)_{m_{1}} \ldots\left(\gamma_{r}\right)_{m_{r}}} & \frac{\left(x_{1}\right)^{m_{1}}}{m_{1} !} \ldots \frac{\left(x_{r}\right)^{m_{r}}}{m_{r} !} \\
& \times \frac{(-1)^{\rho\left(m_{1}+\ldots+m_{k}\right)+m_{k+1}+\ldots+m_{r}} t^{n}}{\left(n-\rho\left(m_{1}+\ldots+m_{k}\right)-m_{k+1}-\ldots-m_{r}\right) !},
\end{aligned}
$$

where we have used the relation $[29, \mathrm{p} .102]$

$$
\sum_{n=0}^{\infty} \sum_{k_{1}, \ldots, k_{r}=0}^{m_{1} k_{1}+\ldots+m_{r} k_{r} \leq n} \Phi\left(k_{1}, \ldots, k_{r} ; n\right)=\sum_{n=0}^{\infty} \sum_{k_{1}, \ldots, k_{r}=0}^{\infty} \Phi\left(k_{1}, \ldots, k_{r} ; n+m_{1} k_{1}+\ldots+m_{r} k_{r}\right),
$$

with $n \rightarrow n+\rho\left(m_{1}+\ldots+m_{k}\right)+m_{k+1}+\ldots+m_{r}$ and we get

$$
\begin{aligned}
S=\sum_{n=0}^{\infty} \sum_{m_{1}, \ldots, m_{r}=0}^{\infty}(\lambda)_{n+\rho\left(m_{1}+\ldots+m_{k}\right)+m_{k+1}+\ldots+m_{r}} \frac{\left(\beta_{k+1}\right)_{m_{k+1}} \ldots\left(\beta_{r}\right)_{m_{r}}}{\left(\gamma_{1}\right)_{m_{1}} \ldots\left(\gamma_{r}\right)_{m_{r}}} \frac{\left(x_{1}\right)^{m_{1}}}{m_{1} !} \ldots \frac{\left(x_{r}\right)^{m_{r}}}{m_{r} !} \\
\times \frac{(-1)^{\rho\left(m_{1}+\ldots+m_{k}\right)+m_{k+1}+\ldots+m_{r}} t^{n+\rho\left(m_{1}+\ldots+m_{k}\right)+m_{k+1}+\ldots+m_{r}}}{n !} .
\end{aligned}
$$

Using (2.5) with $k=\rho\left(m_{1}+\ldots+m_{k}\right)+m_{k+1}+\ldots+m_{r}$, we have

$$
(\lambda)_{n+\rho\left(m_{1}+\ldots+m_{k}\right)+m_{k+1}+\ldots+m_{r}}=(\lambda)_{\rho\left(m_{1}+\ldots+m_{k}\right)+\ldots+m_{k+1}+\ldots+m_{r}}\left(\lambda+\rho\left(m_{1}+\ldots+m_{k}\right)+m_{k+1}+\ldots+m_{r}\right)_{n},
$$


and we can write (2.14)

$$
\begin{aligned}
S=\sum_{m_{1}, \ldots, m_{r}=0}^{\infty}(\lambda)_{\rho\left(m_{1}+\ldots+m_{k}\right)+\ldots+m_{k+1}+\ldots+m_{r}} & \frac{\left(\beta_{k+1}\right)_{m_{k+1}} \ldots\left(\beta_{r}\right)_{m_{r}}}{\left(\gamma_{1}\right)_{m_{1}} \ldots\left(\gamma_{r}\right)_{m_{r}}} \\
& \times \frac{\left[x_{1}(-t)^{\rho}\right]^{m_{1}} \ldots\left[x_{k}(-t)^{\rho}\right]^{m_{k}}\left[-x_{k+1} t\right]^{m_{k+1}} \ldots\left[-x_{r} t\right]^{m_{r}}}{m_{1} ! \ldots m_{k} ! m_{k+1} ! \ldots m_{r} !} \\
& \times \sum_{n=0}^{\infty} \frac{\left(\lambda+\rho\left(m_{1}+\ldots+m_{k}\right)+m_{k+1}+\ldots+m_{r}\right)_{n}}{n !} t^{n},
\end{aligned}
$$

where using (2.8) and we have

$$
\begin{aligned}
& S=(1-t)^{-\lambda} \sum_{m_{1}, \ldots, m_{r}=0}^{\infty}(\lambda)_{\rho\left(m_{1}+\ldots+m_{k}\right)+\ldots+m_{k+1}+\ldots+m_{r}} \frac{\left(\beta_{k+1}\right)_{m_{k+1}} \ldots\left(\beta_{r}\right)_{m_{r}}}{\left(\gamma_{1}\right)_{m_{1}} \ldots\left(\gamma_{r}\right)_{m_{r}}} \\
& \times\left[\frac{x_{1}(-t)^{\rho}}{(1-t)^{\rho}}\right]^{m_{1}} \cdots\left[\frac{x_{k}(-t)^{\rho}}{(1-t)^{\rho}}\right]^{m_{k}}\left[\frac{-x_{k+1} t}{(1-t)}\right]^{m_{k+1}} \ldots\left[\frac{-x_{r} t}{(1-t)}\right]^{m_{r}} \frac{1}{m_{1} !} \ldots \frac{1}{m_{r} !} \\
& =(1-t)^{-\lambda(k)} E^{(r)}\left(\lambda, \beta_{k+1}, \ldots, \beta_{r} ; \gamma_{1}, \ldots, \gamma_{r} ; \frac{x_{1}(-t)^{\rho}}{(1-t)^{\rho}}, \ldots, \frac{x_{k}(-t)^{\rho}}{(1-t)^{\rho}}, \frac{-x_{k+1} t}{(1-t)}, \ldots, \frac{-x_{r} t}{(1-t)}\right)
\end{aligned}
$$

which completes the proof.

Theorem 2.9. We have the following generating function for the multivariable multiple hypergeometric functions ${ }^{(k)} E^{(r)}$ defined by (2.1):

$$
\begin{array}{r}
\sum_{n=0}^{\infty}{ }^{(k)} E^{(r)}\left(-n, \beta_{k+1}, \ldots, \beta_{r} ; \gamma_{1}, \ldots, \gamma_{r} ; x_{1}, \ldots, x_{r}\right) \frac{t^{n}}{n !}=e_{0}^{t} F_{1}\left(-, \gamma_{1} ; x_{1}(-t)^{\rho}\right) \ldots{ }_{0} F_{1}\left(-, \gamma_{k} ; x_{k}(-t)^{\rho}\right) \\
\times \Phi\left(\beta_{k+1}, \gamma_{k+1} ;-x_{k+1} t\right) \ldots \Phi\left(\beta_{r}, \gamma_{r} ;-x_{r} t\right)
\end{array}
$$

where $|t|<1$ and $\Phi$ is confluent hypergeometric function and ${ }_{0} F_{1}$ is hypergeometric series, given by (1.3) and (1.4), respectively.

Proof. Let $S$ denote the first member of assertion (2.15). Then,

$$
\begin{aligned}
S & =\sum_{n=0}^{\infty}{ }^{(k)} E^{(r)}\left(-n, \beta_{k+1}, \ldots, \beta_{r} ; \gamma_{1}, \ldots, \gamma_{r} ; x_{1}, \ldots, x_{r}\right) \frac{t^{n}}{n !} \\
& =\sum_{n=0}^{\infty} \sum_{m_{1}, \ldots, m_{r}=0}^{\rho\left(m_{1}+\ldots+m_{k}\right)+m_{k+1}+\ldots+m_{r} \leq n}(-n)_{\rho\left(m_{1}+\ldots+m_{k}\right)+m_{k+1}+\ldots+m_{r}} \frac{\left(\beta_{k+1}\right)_{m_{k+1}} \ldots\left(\beta_{r}\right)_{m_{r}}}{\left(\gamma_{1}\right)_{m_{1}} \ldots\left(\gamma_{r}\right)_{m_{r}}} \frac{\left(x_{1}\right)^{m_{1}}}{m_{1} !} \ldots \frac{\left(x_{r}\right)^{m_{r}}}{m_{r} !} \frac{t^{n}}{n !}
\end{aligned}
$$

and using (2.12)

$$
\begin{aligned}
S=\sum_{n=0}^{\infty} \sum_{m_{1}, \ldots, m_{r}=0}^{\rho\left(m_{1}+\ldots+m_{k}\right)+m_{k+1}+\ldots+m_{r} \leq n} \frac{\left(\beta_{k+1}\right)_{m_{k+1}} \ldots\left(\beta_{r}\right)_{m_{r}}}{\left(\gamma_{1}\right)_{m_{1}} \ldots\left(\gamma_{r}\right)_{m_{r}}} \frac{\left(x_{1}\right)^{m_{1}}}{m_{1} !} \ldots \frac{\left(x_{r}\right)^{m_{r}}}{m_{r} !} \\
\times \frac{(-1)^{\rho\left(m_{1}+\ldots+m_{k}\right)+m_{k+1}+\ldots+m_{r}} t^{n}}{\left(n-\rho\left(m_{1}+\ldots+m_{k}\right)-m_{k+1}-\ldots-m_{r}\right) !}
\end{aligned}
$$


and using (2.13) with $n \rightarrow n+\rho\left(m_{1}+\ldots+m_{k}\right)+m_{k+1}+\ldots+m_{r}$ and we have

$$
\begin{aligned}
S & =\sum_{n=0}^{\infty} \sum_{m_{1}, \ldots, m_{r}=0}^{\infty} \frac{\left(\beta_{k+1}\right)_{m_{k+1}} \ldots\left(\beta_{r}\right)_{m_{r}}}{\left(\gamma_{1}\right)_{m_{1}} \ldots\left(\gamma_{r}\right)_{m_{r}}} \frac{\left[x_{1}(-t)^{\rho}\right]^{m_{1}} \ldots\left[x_{k}(-t)^{\rho}\right]^{m_{k}}\left[-x_{k+1} t\right]^{m_{k+1}} \ldots\left[-x_{r} t\right]^{m_{r}}}{m_{1} ! \ldots m_{k} ! m_{k+1} ! \ldots m_{r} !} \sum_{n=0}^{\infty} \frac{t^{n}}{n !} \\
= & e^{t} \sum_{m_{1}, \ldots, m_{r}=0}^{\infty} \frac{\left(\beta_{k+1}\right)_{m_{k+1}} \ldots\left(\beta_{r}\right)_{m_{r}}}{\left(\gamma_{1}\right)_{m_{1}} \ldots\left(\gamma_{r}\right)_{m_{r}}}\left[x_{1}(-t)^{\rho}\right]^{m_{1}} \ldots\left[x_{k}(-t)^{\rho}\right]^{m_{k}}\left[-x_{k+1} t\right]^{m_{k+1}} \ldots\left[-x_{r} t\right]^{m_{r}} \frac{1}{m_{1} !} \ldots \frac{1}{m_{r} !} \\
= & e^{t} \sum_{m_{1}=0}^{\infty} \frac{1}{\left(\gamma_{1}\right)_{m_{1}}}\left[x_{1}(-t)^{\rho}\right]^{m_{1}} \frac{1}{m_{1} !} \ldots \sum_{m_{k}=0}^{\infty} \frac{1}{\left(\gamma_{1}\right)_{m_{k}}}\left[x_{k}(-t)^{\rho}\right]^{m_{k}} \frac{1}{m_{k} !} \\
& \times \sum_{m_{k+1}=0}^{\infty} \frac{\left(\beta_{k+1}\right)_{m_{k+1}}}{\left(\gamma_{1}\right)_{m_{k+1}}}\left[-x_{k+1} t\right]^{m_{k+1}} \frac{1}{m_{k+1} !} \ldots \sum_{m_{r}=0}^{\infty} \frac{\left(\beta_{r}\right)_{m_{r}}}{\left(\gamma_{r}\right)_{m_{r}}}\left[-x_{r} t\right]^{m_{r}} \frac{1}{m_{r} !}
\end{aligned}
$$

If the hypergeometric series in (1.4) and confluent hypergeometric function in (1.3) are used,

$$
S=e_{0}^{t} F_{1}\left(-, \gamma_{1} ; x_{1}(-t)^{\rho}\right) \ldots 0 F_{1}\left(-, \gamma_{k} ; x_{k}(-t)^{\rho}\right) \Phi\left(\beta_{k+1}, \gamma_{k+1} ;-x_{k+1} t\right) \ldots \Phi\left(\beta_{r}, \gamma_{r} ;-x_{r} t\right),
$$

which completes the proof.

Lemma 2.10. The following relation is provided for the Pochhammer symbol given by (1.2)

$$
(1-\lambda-n)_{m_{1}}=\frac{\left(\begin{array}{c}
\lambda+n-1 \\
n
\end{array}\right)(1-\lambda)_{m_{1}}}{\left(\begin{array}{c}
\lambda-m_{1}+n-1 \\
n
\end{array}\right)}
$$

Proof. On the left side of Equation 2.16, if the definition of the Pochhammer symbol is applied, we get

$$
\begin{aligned}
& (1-\lambda-n)_{m_{1}}=(1-\lambda-n)(2-\lambda-n) \ldots\left(1-\lambda-n+m_{1}-1\right) \\
& =(1-\lambda-n)(2-\lambda-n) \ldots\left(m_{1}-\lambda-n\right) \\
& =\frac{(-1)^{m_{1}}(\lambda+n-1)(\lambda+n-2) \ldots\left(\lambda+n-m_{1}\right)\left(\lambda+n-m_{1}-1\right) !}{\left(\lambda+n-m_{1}-1\right) !} \\
& =\frac{(-1)^{m_{1}}(\lambda+n-1) ! n !\left(\lambda-m_{1}-1\right) !(\lambda-1) !}{\left(\lambda+n-m_{1}-1\right) ! n !\left(\lambda-m_{1}-1\right) !(\lambda-1) !} \\
& =\frac{\left(\begin{array}{c}
\lambda+n-1 \\
n
\end{array}\right)(\lambda-1) !(-1)^{m_{1}}}{\left(\begin{array}{c}
\lambda-m_{1}+n-1 \\
n
\end{array}\right)\left(\lambda-m_{1}-1\right) !} \\
& =\frac{\left(\begin{array}{c}
\lambda+n-1 \\
n
\end{array}\right)(\lambda-1)(\lambda-2) \ldots\left(\lambda-1-\left(m_{1}-1\right)\right)\left(\lambda-1-m_{1}\right) !(-1)^{m_{1}}}{\left(\begin{array}{c}
\lambda-m_{1}+n-1 \\
n
\end{array}\right)\left(\lambda-m_{1}-1\right) !} \\
& =\frac{\left(\begin{array}{c}
\lambda+n-1 \\
n
\end{array}\right)(1-\lambda)(2-\lambda) \ldots\left(1-\lambda+m_{1}-1\right)}{\left(\begin{array}{c}
\lambda-m_{1}+n-1 \\
n
\end{array}\right)} \\
& =\frac{\left(\begin{array}{c}
\lambda+n-1 \\
n
\end{array}\right)(1-\lambda)_{m_{1}}}{\left(\begin{array}{c}
\lambda-m_{1}+n-1 \\
n
\end{array}\right)}
\end{aligned}
$$

which completes the proof. 
Theorem 2.11. We have the following generating function for the multivariable multiple hypergeometric functions ${ }^{(k)} E^{(r)}$ defined by $(2.1)$ :

$$
\begin{aligned}
\sum_{n=0}^{\infty}\left(\begin{array}{c}
\lambda+n-1 \\
n
\end{array}\right){ }^{(k)} E^{(r)}\left(\alpha, \beta_{k+1}, \ldots, \beta_{r} ; 1-\lambda-n, \gamma_{2}, \ldots, \gamma_{r} ; x_{1}, \ldots, x_{r}\right) t^{n} & \\
= & (1-t)^{-\lambda(k)} E^{(r)}\left(\alpha, \beta_{k+1}, \ldots, \beta_{r} ; 1-\lambda, \gamma_{2}, \ldots, \gamma_{r} ; x_{1}(1-t), x_{2}, \ldots, x_{r}\right),
\end{aligned}
$$

where $\lambda \in \mathbb{C},|t|<1$.

Proof. Let $T$ denote the first member of assertion (2.17). Then,

$$
\begin{aligned}
T=\sum_{n=0}^{\infty} \sum_{m_{1}, \ldots, m_{r}=0}^{\infty}\left(\begin{array}{c}
\lambda+n-1 \\
n
\end{array}\right) & (\alpha)_{\rho\left(m_{1}+\ldots+m_{k}\right)+m_{k+1}+\ldots+m_{r}} \\
& \times \frac{\left(\beta_{k+1}\right)_{m_{k+1}} \ldots\left(\beta_{r}\right)_{m_{r}}}{(1-\lambda-n)_{m_{1}}\left(\gamma_{2}\right)_{m_{2}} \ldots\left(\gamma_{r}\right)_{m_{r}}} \frac{\left(x_{1}\right)^{m_{1}}}{m_{1} !} \ldots \frac{\left(x_{r}\right)^{m_{r}}}{m_{r} !} t^{n} .
\end{aligned}
$$

Using the Lemma 2.10

$$
\begin{aligned}
T=\sum_{n=0}^{\infty} \sum_{m_{1}, \ldots, m_{r}=0}^{\infty}\left(\begin{array}{c}
\lambda-m_{1}+n-1 \\
n
\end{array}\right)(\alpha)_{\rho\left(m_{1}+\ldots+m_{k}\right)+m_{k+1}+\ldots+m_{r}} \\
\quad \times \frac{\left(\beta_{k+1}\right)_{m_{k+1} \ldots\left(\beta_{r}\right)_{m_{r}}}}{(1-\lambda)_{m_{1}}\left(\gamma_{2}\right)_{m_{2}} \ldots\left(\gamma_{r}\right)_{m_{r}}} \frac{\left(x_{1}\right)^{m_{1}}}{m_{1} !} \ldots \frac{\left(x_{r}\right)^{m_{r}}}{m_{r} !} t^{n} \\
=\sum_{n=0}^{\infty} \sum_{m_{1}, \ldots, m_{r}=0}^{\infty} \frac{\left(\lambda-m_{1}+n-1\right) !}{\left(\lambda-m_{1}-1\right) !}(\alpha)_{\rho\left(m_{1}+\ldots+m_{k}\right)+m_{k+1}+\ldots+m_{r}} \\
\times \frac{\left(\beta_{k+1}\right)_{m_{k+1}} \ldots\left(\beta_{r}\right)_{m_{r}}}{(1-\lambda)_{m_{1}}\left(\gamma_{2}\right)_{m_{2}} \ldots\left(\gamma_{r}\right)_{m_{r}}} \frac{\left(x_{1}\right)^{m_{1}}}{m_{1} !} \ldots \frac{\left(x_{r}\right)^{m_{r}}}{m_{r} !} \frac{t^{n}}{n !} .
\end{aligned}
$$

Using the idendity

$$
\Gamma(n)=(n-1) !,
$$

and the definition of Pochhammer symbol given by (1.2), we have

$$
\frac{\left(\lambda-m_{1}+n-1\right) !}{\left(\lambda-m_{1}-1\right) !}=\frac{\Gamma\left(\lambda-m_{1}+n\right)}{\Gamma\left(\lambda-m_{1}\right)}=\left(\lambda-m_{1}\right)_{n} .
$$

Thus, we can rewrite (2.18)

$$
T=\sum_{n=0}^{\infty} \sum_{m_{1}, \ldots, m_{r}=0}^{\infty}(\alpha)_{\rho\left(m_{1}+\ldots+m_{k}\right)+m_{k+1}+\ldots+m_{r}} \frac{\left(\beta_{k+1}\right)_{m_{k+1}} \ldots\left(\beta_{r}\right)_{m_{r}}}{(1-\lambda)_{m_{1}}\left(\gamma_{2}\right)_{m_{2}} \ldots\left(\gamma_{r}\right)_{m_{r}}} \frac{\left(x_{1}\right)^{m_{1}}}{m_{1} !} \ldots \frac{\left(x_{r}\right)^{m_{r}}}{m_{r} !} \sum_{n=0}^{\infty} \frac{\left(\lambda-m_{1}\right)_{n}}{n !} t^{n} .
$$

Using the equation (2.7) with $c=\lambda-m_{1}$, we get

$$
\begin{aligned}
T & =(1-t)^{-\lambda} \sum_{m_{1}, \ldots, m_{r}=0}^{\infty}(\alpha)_{\rho\left(m_{1}+\ldots+m_{k}\right)+m_{k+1}+\ldots+m_{r}} \frac{\left(\beta_{k+1}\right)_{m_{k+1}} \ldots\left(\beta_{r}\right)_{m_{r}}}{(1-\lambda)_{m_{1}}\left(\gamma_{2}\right)_{m_{2}} \ldots\left(\gamma_{r}\right)_{m_{r}}} \frac{\left[x_{1}(1-t)\right]^{m_{1}}}{m_{1} !} \ldots \frac{\left[x_{r}\right]^{m_{r}}}{m_{r} !} \\
& =(1-t)^{-\lambda(k)} E^{(r)}\left(\alpha, \beta_{k+1}, \ldots, \beta_{r} ; 1-\lambda, \gamma_{2}, \ldots, \gamma_{r} ; x_{1}(1-t), x_{2}, \ldots, x_{r}\right),
\end{aligned}
$$

which completes the proof.

In the next theorem, let $\Psi_{m}$ denote the following special functions ${ }^{(k)} E^{(r)}$.

$$
\Psi_{m}={ }^{(k)} E^{(r)}\left(\alpha, \beta_{k+1}, \ldots, \beta_{r} ; 1-\lambda-m, \gamma_{2}, \ldots, \gamma_{r} ; x_{1}, \ldots, x_{r}\right) .
$$

Theorem 2.12. The following generating function for the multivariable multiple hypergeometric functions $\Psi_{m}$ holds true:

$$
\sum_{n=0}^{\infty}\left(\begin{array}{c}
\lambda+m+n-1 \\
n
\end{array}\right) \Psi_{m+n}\left(x_{1}, \ldots, x_{r}\right) t^{n}=(1-t)^{-\lambda-m} \Psi_{m}\left(x_{1}(1-t), x_{2}, \ldots, x_{r}\right)
$$


Proof. Let $T$ denote the first member of assertion (2.19). Then,

$$
\begin{aligned}
T & =\sum_{n=0}^{\infty}\left(\begin{array}{c}
\lambda+m+n-1 \\
n
\end{array}\right) \Psi_{m+n}\left(x_{1}, \ldots, x_{r}\right) t^{n} \\
& =\sum_{n=0}^{\infty}\left(\begin{array}{c}
\lambda+m+n-1 \\
n
\end{array}\right){ }^{(k)} E^{(r)}\left(\alpha, \beta_{k+1}, \ldots, \beta_{r} ; 1-\lambda-m-n, \gamma_{2}, \ldots, \gamma_{r} ; x_{1}, \ldots, x_{r}\right) t^{n}
\end{aligned}
$$

From Theorem 2.11,

$$
\begin{aligned}
T & =(1-t)^{-\lambda-m(k)} E^{(r)}\left(\alpha, \beta_{k+1}, \ldots, \beta_{r} ; 1-\lambda-m, \gamma_{2}, \ldots, \gamma_{r} ; x_{1}(1-t), x_{2}, \ldots, x_{r}\right) \\
& =(1-t)^{-\lambda-m} \Psi_{m}\left(x_{1}(1-t), x_{2}, \ldots, x_{r}\right),
\end{aligned}
$$

which completes the proof.

Similar to Corollaries 2.3, 2.4 and Remarks 2.5, 2.6 and 2.7, we also get the following corollary for Theorems 2.8, 2.9, 2.11 and 2.12 .

Corollary 2.13. If

(1) $k=1, r=2$

(2) $\rho=2$

(3) $\rho=2, k=1$ and $r=2$

(4) $\rho=2, k=0$

(5) $\rho=2, k=0$ and $r=2$

are taken in all Teorems 2.8, 2.9, 2.11 and 2.12, generating function relations are found for the functions of twovariable multiple hypergeometric, fourth kind multivariable Horn, fourth kind Horn, first kind Lauricella and second kind Appell given by (2.2), (1.8), (1.7), (1.6) and (1.5), respectively.

\section{Multilinear and Multilateral Generating Functions}

In this section, we derive several families of multilinear and multilateral generating function for multivariable multiple hypergeometric functions defined by $(2.1)$ by using the similar method considered in $[11,19,20]$.

Theorem 3.1. Corresponding to an identically non-vanishing function $\Omega_{\mu}\left(y_{1}, \ldots, y_{s}\right)$ of $s$ complex variables $y_{1}, \ldots, y_{s}$ $(s \in N)$ and of complex order $\mu$, let

$$
\Lambda_{\mu, \psi}\left(y_{1}, \ldots, y_{s} ; \zeta\right):=\sum_{k=0}^{\infty} a_{k} \Omega_{\mu+\psi k}\left(y_{1}, \ldots, y_{s}\right) \zeta^{k},
$$

where $a_{k} \neq 0, \mu, \psi \in C$ and

$$
\begin{aligned}
& \Theta_{n, p}^{\mu, \psi}\left(x_{1}, \ldots, x_{r} ; y_{1}, \ldots, y_{s} ; \xi\right) \\
& \quad:=\sum_{k=0}^{[n / p]} a_{k}(\lambda)_{n-p k}{ }^{(k)} E^{(r)}\left(\lambda+n-p k, \beta_{k+1}, \ldots, \beta_{r} ; \gamma_{1}, \ldots, \gamma_{r} ; x_{1}, \ldots, x_{r}\right) \Omega_{\mu+\psi k}\left(y_{1}, \ldots, y_{s}\right) \frac{\xi^{k}}{(n-p k) !} .
\end{aligned}
$$

Then, for $p \in N$, we have

$$
\begin{aligned}
\sum_{n=0}^{\infty} \Theta_{n, p}^{\mu, \psi} & \left(x_{1}, \ldots, x_{r} ; y_{1}, \ldots, y_{s} ; \frac{\eta}{t^{p}}\right) t^{n}=\Lambda_{\mu, \psi}\left(y_{1}, \ldots, y_{s} ; \eta\right)(1-t)^{-\lambda} \\
& \times{ }^{(k)} E^{(r)}\left(\lambda, \beta_{k+1}, \ldots, \beta_{r} ; \gamma_{1}, \ldots, \gamma_{r} ; \frac{x_{1}}{(1-t)^{\rho}}, \ldots, \frac{x_{k}}{(1-t)^{\rho}}, \frac{x_{k+1}}{(1-t)}, \ldots, \frac{x_{r}}{(1-t)}\right),
\end{aligned}
$$

provided that each member of (3.1) exists. 
Proof. For convenience, let $S$ denote the first member of the assertion (3.1). Then,

$$
S=\sum_{n=0}^{\infty} \sum_{k=0}^{[n / p]} a_{k}(\lambda)_{n-p k}{ }^{(k)} E^{(r)}\left(\lambda+n-p k, \beta_{k+1}, \ldots, \beta_{r} ; \gamma_{1}, \ldots, \gamma_{r} ; x_{1}, \ldots, x_{r}\right) \Omega_{\mu+\psi k}\left(y_{1}, \ldots, y_{s}\right) \frac{\eta^{k} t^{n-p k}}{(n-p k) !} .
$$

Replacing $n$ by $n+p k$, we may write that

$$
\begin{aligned}
& S=\sum_{n=0}^{\infty} \sum_{k=0}^{\infty} a_{k}(\lambda)_{n}{ }^{(k)} E^{(r)}\left(\lambda+n, \beta_{k+1}, \ldots, \beta_{r} ; \gamma_{1}, \ldots, \gamma_{r} ; x_{1}, \ldots, x_{r}\right) \Omega_{\mu+\psi k}\left(y_{1}, \ldots, y_{s}\right) \eta^{k} \frac{t^{n}}{n !} \\
& =\sum_{n=0}^{\infty}(\lambda)_{n}{ }^{(k)} E^{(r)}\left(\lambda+n, \beta_{k+1}, \ldots, \beta_{r} ; \gamma_{1}, \ldots, \gamma_{r} ; x_{1}, \ldots, x_{r}\right) \frac{t^{n}}{n !} \sum_{k=0}^{\infty} a_{k} \Omega_{\mu+\psi k}\left(y_{1}, \ldots, y_{s}\right) \eta^{k} \\
& =(1-t)^{-\lambda(k)} E^{(r)}\left(\lambda, \beta_{k+1}, \ldots, \beta_{r} ; \gamma_{1}, \ldots, \gamma_{r} ; \frac{x_{1}}{(1-t)^{\rho}}, \ldots, \frac{x_{k}}{(1-t)^{\rho}}, \frac{x_{k+1}}{(1-t)}, \ldots, \frac{x_{r}}{(1-t)}\right) \Lambda_{\mu, \psi}\left(y_{1}, \ldots, y_{s} ; \eta\right),
\end{aligned}
$$

which completes the proof.

In a similar manner, we also get the Theorem 3.2 and 3.3 immediately.

Theorem 3.2. Corresponding to an identically non-vanishing function $\Omega_{\mu}\left(y_{1}, \ldots, y_{s}\right)$ of $s$ complex variables $y_{1}, \ldots, y_{s}$ $(s \in N)$ and of complex order $\mu$, let

$$
\Lambda_{\mu, \psi}\left(y_{1}, \ldots, y_{s} ; \zeta\right):=\sum_{k=0}^{\infty} a_{k} \Omega_{\mu+\psi k}\left(y_{1}, \ldots, y_{s}\right) \zeta^{k}
$$

where $a_{k} \neq 0, \mu, \psi \in C$ and

$$
\begin{aligned}
& \Theta_{n, p}^{\mu, \psi}\left(x_{1}, \ldots, x_{r} ; y_{1}, \ldots, y_{s} ; \xi\right) \\
& \quad:=\sum_{k=0}^{[n / p]} a_{k}(\lambda)_{n-p k}{ }^{(k)} E^{(r)}\left(-n-p k, \beta_{k+1}, \ldots, \beta_{r} ; \gamma_{1}, \ldots, \gamma_{r} ; x_{1}, \ldots, x_{r}\right) \Omega_{\mu+\psi k}\left(y_{1}, \ldots, y_{s}\right) \frac{\xi^{k}}{(n-p k) !} .
\end{aligned}
$$

Then, for $p \in N$, we have

$$
\begin{aligned}
& \sum_{n=0}^{\infty} \Theta_{n, p}^{\mu, \psi}\left(x_{1}, \ldots, x_{r} ; y_{1}, \ldots, y_{s} ; \frac{\eta}{t^{p}}\right) t^{n}=\Lambda_{\mu, \psi}\left(y_{1}, \ldots, y_{s} ; \eta\right)(1-t)^{-\lambda} \\
& \quad \times{ }^{(k)} E^{(r)}\left(\lambda, \beta_{k+1}, \ldots, \beta_{r} ; \gamma_{1}, \ldots, \gamma_{r} ; \frac{x_{1}(-t)^{\rho}}{(1-t)^{\rho}}, \ldots, \frac{x_{k}(-t)^{\rho}}{(1-t)^{\rho}}, \frac{-x_{k+1} t}{(1-t)}, \ldots, \frac{-x_{r} t}{(1-t)}\right),
\end{aligned}
$$

provided that each member of (3.2) exists.

Proof. By using similar method in the proof of Theorem 3.1, we arrive at the desired result.

Theorem 3.3. Corresponding to an identically non-vanishing function $\Omega_{\mu}\left(y_{1}, \ldots, y_{s}\right)$ of $s$ complex variables $y_{1}, \ldots, y_{s}$ $(s \in N)$ and of complex order $\mu$, let

$$
\Lambda_{\mu, \psi}\left(y_{1}, \ldots, y_{s} ; \zeta\right):=\sum_{k=0}^{\infty} a_{k} \Omega_{\mu+\psi k}\left(y_{1}, \ldots, y_{s}\right) \zeta^{k},
$$

where $a_{k} \neq 0, \mu, \psi \in C$ and

$$
\begin{gathered}
\Theta_{n, p}^{\mu, \psi}\left(x_{1}, \ldots, x_{r} ; y_{1}, \ldots, y_{s} ; \xi\right):=\sum_{k=0}^{[n / p]} a_{k}^{(k)} E^{(r)}\left(-n-p k, \beta_{k+1}, \ldots, \beta_{r} ; \gamma_{1}, \ldots, \gamma_{r} ; x_{1}, \ldots, x_{r}\right) \\
\times \Omega_{\mu+\psi k}\left(y_{1}, \ldots, y_{s}\right) \frac{\xi^{k}}{(n-p k) !} .
\end{gathered}
$$


Then, for $p \in N$, we have

$$
\begin{array}{r}
\sum_{n=0}^{\infty} \Theta_{n, p}^{\mu, \psi}\left(x_{1}, \ldots, x_{r} ; y_{1}, \ldots, y_{s} ; \frac{\eta}{t^{p}}\right) t^{n}=\Lambda_{\mu, \psi}\left(y_{1}, \ldots, y_{s} ; \eta\right) e_{0}^{t} F_{1}\left(-, \gamma_{1} ; x_{1}(-t)^{\rho}\right) \ldots{ }_{0} F_{1}\left(-, \gamma_{k} ; x_{k}(-t)^{\rho}\right) \\
\times \Phi\left(\beta_{k+1}, \gamma_{k+1} ;-x_{k+1} t\right) \ldots \Phi\left(\beta_{r}, \gamma_{r} ;-x_{r} t\right)
\end{array}
$$

provided that each member of (3.3) exists.

Proof. We get the required result by using the generating functions in Theorem 2.9 and the techniques used to prove Theorem 3.1.

Theorem 3.4. Corresponding to an identically non-vanishing function $\Omega_{\mu}\left(y_{1}, \ldots, y_{s}\right)$ of $r$ complex variables $y_{1}, \ldots, y_{s}$ $(r \in N)$ and of complex order $\mu$, let

$$
\Lambda_{m, q}\left(x_{1}, \ldots, x_{r} ; y_{1}, \ldots, y_{s} ; t\right):=\sum_{n=0}^{\infty} a_{n} \Psi_{m+n q}\left(x_{1}, \ldots, x_{r}\right) \Omega_{\mu+p n}\left(y_{1}, \ldots, y_{s}\right) t^{n},
$$

where $\left(a_{n} \neq 0, \mu \in C\right), \Psi_{m}$ is defined by (2.7) and

$$
N_{n, m, q}^{p, \mu}\left(y_{1}, \ldots, y_{s} ; z\right):=\sum_{k=0}^{[n / q]} a_{k} \Omega_{\mu+p k}\left(y_{1}, \ldots, y_{s}\right) z^{k} .
$$

Then, for every nonnegative integer $m$,

$$
\begin{aligned}
& \sum_{n=0}^{\infty}\left(\begin{array}{c}
\lambda+m+n-1 \\
n
\end{array}\right) \Psi_{m+n}\left(x_{1}, \ldots, x_{r}\right) N_{n, m, q}^{p, \mu}\left(y_{1}, \ldots, y_{s} ; z\right) t^{n} \\
& \quad=(1-t)^{-\lambda-m} \Lambda_{m, q}\left(x_{1}(1-t), x_{2}, \ldots, x_{r} ; y_{1}, \ldots, y_{s} ; \frac{z t^{q}}{(1-t)^{q}}\right)
\end{aligned}
$$

provided that each member of (3.4) exists.

Proof. Let $T$ denote the left-hand side of equality (3.4). Then we have

$$
\begin{aligned}
T & =\sum_{n=0}^{\infty}\left(\begin{array}{c}
\lambda+m+n-1 \\
n
\end{array}\right) \Psi_{m+n}\left(x_{1}, \ldots, x_{r}\right) \sum_{k=0}^{[n / q]} a_{k} \Omega_{\mu+p k}\left(y_{1}, \ldots, y_{s}\right) z^{k} t^{n} \\
& =\sum_{k=0}^{\infty}\left(\sum_{n=0}^{\infty}\left(\begin{array}{c}
\lambda+m+n+q k-1 \\
n
\end{array}\right) \Psi_{m+n+q k}\left(x_{1}, \ldots, x_{r}\right) t^{n}\right) a_{k} \Omega_{\mu+p k}\left(y_{1}, \ldots, y_{s}\right)\left(z t^{q}\right)^{k} \\
& =\sum_{k=0}^{\infty}(1-t)^{-\lambda-m-q k} \Psi_{m+q k}\left(x_{1}(1-t), x_{2}, \ldots, x_{r}\right) a_{k} \Omega_{\mu+p k}\left(y_{1}, \ldots, y_{s}\right)\left(z t^{q}\right)^{k} \\
& =(1-t)^{-\lambda-m} \sum_{k=0}^{\infty} a_{k} \Psi_{m+q k}\left(x_{1}(1-t), x_{2}, \ldots, x_{r}\right) \Omega_{\mu+p k}\left(y_{1}, \ldots, y_{s}\right)\left(\frac{z t^{q}}{(1-t)^{q}}\right)^{k} \\
& =(1-t)^{-\lambda-m} \Lambda_{m, q}\left(x_{1}(1-t), x_{2}, \ldots, x_{r} ; y_{1}, \ldots, y_{s} ; \frac{z t^{q}}{(1-t)^{q}}\right)
\end{aligned}
$$

which completes the proof.

\section{Special Cases}

As an application of the above Theorems, when the multivariable function $\Omega_{\mu+\psi k}\left(y_{1}, \ldots, y_{s}\right), k \in N_{0}, s \in N$, is expressed in terms of simpler functions of one and more variables, then we can give further applications of the above theorems. We first set

$$
s=r, \quad \Omega_{\mu+\psi k}\left(y_{1}, \ldots, y_{r}\right)=u_{\mu+\psi k}^{\left(\alpha_{1}, \ldots, \alpha_{r}\right)}\left(y_{1}, \ldots, y_{r}\right)
$$


in Theorem 3.1, where the Erkus-Srivastava polynomials $u_{n}^{\left(\alpha_{1}, \ldots, \alpha_{r}\right)}\left(x_{1}, \ldots, x_{r}\right)$, generated by [11]

$$
\sum_{n=0}^{\infty} u_{n}^{\left(\alpha_{1}, \ldots, \alpha_{r}\right)}\left(x_{1}, \ldots, x_{r}\right) \frac{t^{n}}{n !}=\prod_{j=1}^{r}\left\{\left(1-x_{j} t^{m_{j}}\right)^{-\alpha_{j}}\right\} .
$$

We are thus led to the following result which provides a class of bilateral generating functions for the ${ }^{(k)} E^{(r)}$ multivariable multiple hypergeometric functions and Erkus-Srivastava polynomials respectively defined by (2.1) and generated by (4.1).

Corollary 4.1. If

$$
\begin{gathered}
\Lambda_{\mu, \psi}\left(y_{1}, \ldots, y_{r} ; \zeta\right):=\sum_{k=0}^{\infty} a_{k} u_{\mu+\psi k}^{\left(\alpha_{1}, \ldots, \alpha_{r}\right)}\left(y_{1}, \ldots, y_{r}\right) \zeta^{k} \\
\left(a_{k} \neq 0, \mu, \psi \in \mathbb{C}\right)
\end{gathered}
$$

then, we have

$$
\begin{aligned}
& \sum_{n=0}^{\infty} \sum_{k=0}^{[n / p]} a_{k}(\lambda)_{n-p k}{ }^{(k)} E^{(r)}\left(\lambda+n-p k, \beta_{k+1}, \ldots, \beta_{r} ; \gamma_{1}, \ldots, \gamma_{r} ; x_{1}, \ldots, x_{r}\right) \\
& \quad \times u_{\mu+\psi k}^{\left(\alpha_{1}, \ldots, \alpha_{r}\right)}\left(y_{1}, \ldots, y_{r}\right) \frac{\eta^{k}}{t^{p k}} \frac{t^{n}}{(n-p k) !} \\
& \quad={ }^{(k)} E^{(r)}\left(\lambda, \beta_{k+1}, \ldots, \beta_{r} ; \gamma_{1}, \ldots, \gamma_{r} ; \frac{x_{1}}{(1-t)^{\rho}}, \ldots, \frac{x_{k}}{(1-t)^{\rho}}, \frac{x_{k+1}}{(1-t)}, \ldots, \frac{x_{r}}{(1-t)}\right) \\
& \quad \times(1-t)^{-\lambda} \Lambda_{\mu, \psi}\left(y_{1}, \ldots, y_{r} ; \eta\right),
\end{aligned}
$$

provided that each member of (4.2) exists.

Remark 4.2. Using the generating relation (4.1) for Erkus-Srivastava polynomials and getting $a_{k}=1, \mu=0, \psi=1$ in Corollary 4.1, we find that

$$
\begin{aligned}
& \sum_{n=0}^{\infty} \sum_{k=0}^{[n / p]}(\lambda)_{n-p k}{ }^{(k)} E^{(r)}\left(\lambda+n-p k, \beta_{k+1}, \ldots, \beta_{r} ; \gamma_{1}, \ldots, \gamma_{r} ; x_{1}, \ldots, x_{r}\right) \\
& \quad \times u_{k}^{\left(\alpha_{1}, \ldots, \alpha_{r}\right)}\left(y_{1}, \ldots, y_{r}\right) \frac{\eta^{k} t^{n-p k}}{(n-p k) !} \\
& \quad=(1-t)^{-\lambda(k)} E^{(r)}\left(\lambda, \beta_{k+1}, \ldots, \beta_{r} ; \gamma_{1}, \ldots, \gamma_{r} ; \frac{x_{1}}{(1-t)^{\rho}}, \ldots, \frac{x_{k}}{(1-t)^{\rho}}, \frac{x_{k+1}}{(1-t)}, \ldots, \frac{x_{r}}{(1-t)}\right) \\
& \quad \times \prod_{j=1}^{r}\left\{\left(1-y_{i} \eta^{m_{j}}\right)^{-\alpha_{i}}\right\} .
\end{aligned}
$$

If we set

$$
\Omega_{\mu+\psi k}\left(y_{1}, \ldots, y_{r}\right)={ }^{(k)} E^{(r)}\left(\lambda+(\mu+\psi k), \beta_{k+1}, \ldots, \beta_{r} ; \gamma_{1}, \ldots, \gamma_{r} ; x_{1}, \ldots, x_{r}\right)
$$

or

$$
\Omega_{\mu+\psi k}\left(y_{1}, \ldots, y_{r}\right)={ }^{(k)} E^{(r)}\left(-(\mu+\psi k), \beta_{k+1}, \ldots, \beta_{r} ; \gamma_{1}, \ldots, \gamma_{r} ; x_{1}, \ldots, x_{r}\right)
$$

in Theorem 3.1, 3.2 and 3.3, we have bilinear generating function relations for the multivariable multiple hypergeometric functions.

On the other hand, choosing

$$
s=r, \quad \Omega_{\mu+\psi k}\left(y_{1}, y_{2}\right)={ }^{(k)} H_{4}^{(r)}\left(-(\mu+\psi k), \beta_{k+1}, \ldots, \beta_{r} ; \gamma_{1}, \ldots, \gamma_{r} ; x_{1}, \ldots, x_{r}\right)
$$


in Theorem 3.2, where the fourth kind multivariable Horn functions [13], generated by

$$
\begin{aligned}
& \sum_{n=0}^{\infty} \frac{(\lambda)_{n}}{n !}{ }^{(k)} H_{4}^{(r)}\left(-n, \beta_{k+1}, \ldots, \beta_{r} ; \gamma_{1}, \ldots, \gamma_{r} ; x_{1}, \ldots, x_{r}\right) t^{n} \\
& \quad=(1-t)^{-\lambda(k)} H_{4}^{(r)}\left(\lambda, \beta_{k+1}, \ldots, \beta_{r} ; \gamma_{1}, \ldots, \gamma_{r} ; \frac{x_{1} t^{2}}{(1-t)^{2}}, \ldots, \frac{x_{k} t^{2}}{(1-t)^{2}}, \frac{-x_{k+1} t}{(1-t)}, \ldots, \frac{-x_{r} t}{(1-t)}\right),
\end{aligned}
$$

where $|t|<1$.

We are thus led to the following result which provides a class of bilateral generating functions for the multivariable multiple hypergeometric functions ${ }^{(k)} E^{(r)}$ and the fourth kind multivariable Horn functions.

Corollary 4.3. If

$$
\begin{gathered}
\Lambda_{\mu, \psi}\left(y_{1}, \ldots, y_{r} ; \zeta\right):=\sum_{k=0}^{\infty} a_{k}{ }^{(k)} H_{4}^{(r)}\left(-(\mu+\psi k), \beta_{k+1}, \ldots, \beta_{r} ; \gamma_{1}, \ldots, \gamma_{r} ; y_{1}, \ldots, y_{r}\right) \zeta^{k} \\
\left(a_{k} \neq 0, \quad \mu, \psi \in \mathbb{C}\right)
\end{gathered}
$$

then, we have

$$
\begin{aligned}
\sum_{n=0}^{\infty} & \sum_{k=0}^{[n / p]} a_{k}\left(\lambda_{1}\right)_{n-p k}{ }^{(k)} E^{(r)}\left(-n-p k, \beta_{k+1}, \ldots, \beta_{r} ; \gamma_{1}, \ldots, \gamma_{r} ; x_{1}, \ldots, x_{r}\right) \\
& \times{ }^{(k)} H_{4}^{(r)}\left(-(\mu+\psi k), \beta_{k+1}, \ldots, \beta_{r} ; \gamma_{1}, \ldots, \gamma_{r} ; y_{1}, \ldots, y_{r}\right) \frac{\eta^{k} t^{n-p k}}{(n-p k) !} \\
& =(1-t)^{-\lambda_{1}{ }^{(k)}} E^{(r)}\left(\lambda_{1}, \beta_{k+1}, \ldots, \beta_{r} ; \gamma_{1}, \ldots, \gamma_{r} ; \frac{x_{1}(-t)^{\rho}}{(1-t)^{\rho}}, \ldots, \frac{x_{k}(-t)^{\rho}}{(1-t)^{\rho}}, \frac{-x_{k+1} t}{(1-t)}, \ldots, \frac{-x_{r} t}{(1-t)}\right) \\
& \times \Lambda_{\mu, \psi}\left(y_{1}, \ldots, y_{r} ; \eta\right),
\end{aligned}
$$

provided that each member of (4.4) exists.

Remark 4.4. Using the generating relation (4.3) for the fourth kind multivariable Horn functions and getting $a_{k}=\frac{\left(\lambda_{2}\right)_{k}}{k !}$, $\mu=0, \psi=1$, in Corollary 4.3 , we find that

$$
\begin{aligned}
& \sum_{n=0}^{\infty} \sum_{k=0}^{[n / p]}\left(\lambda_{2}\right)_{k}\left(\lambda_{1}\right)_{n-p k}{ }^{(k)} E^{(r)}\left(-n-p k, \beta_{k+1}, \ldots, \beta_{r} ; \gamma_{1}, \ldots, \gamma_{r} ; x_{1}, \ldots, x_{r}\right) \\
& \times{ }^{(k)} H_{4}^{(r)}\left(-k, \beta_{k+1}, \ldots, \beta_{r} ; \gamma_{1}, \ldots, \gamma_{r} ; y_{1}, \ldots, y_{r}\right) \frac{\eta^{k} t^{n-p k}}{k !(n-p k) !} \\
& =(1-t)^{-\lambda_{1}(k)} E^{(r)}\left(\lambda_{1}, \beta_{k+1}, \ldots, \beta_{r} ; \gamma_{1}, \ldots, \gamma_{r} ; \frac{x_{1}(-t)^{\rho}}{(1-t)^{\rho}}, \ldots, \frac{x_{k}(-t)^{\rho}}{(1-t)^{\rho}}, \frac{-x_{k+1} t}{(1-t)}, \ldots, \frac{-x_{r} t}{(1-t)}\right) \\
& \times(1-\eta)^{-\lambda_{2}(k)} H_{4}^{(r)}\left(\lambda_{2}, \beta_{k+1}, \ldots, \beta_{r} ; \gamma_{1}, \ldots, \gamma_{r} ; \frac{y_{1} \eta^{2}}{(1-\eta)^{2}}, \ldots, \frac{y_{k} \eta^{2}}{(1-\eta)^{2}}, \frac{-y_{k+1} \eta}{(1-\eta)}, \ldots, \frac{-y_{r} \eta}{(1-\eta)}\right) \text {. }
\end{aligned}
$$

Remark 4.5. If we take $\rho=2$ in (4.4), we have a bilinear generating function relations for the fourth type multivariable Horn functions.

Furthermore, for every suitable choice of the coefficients $a_{k}\left(k \in N_{0}\right)$, if the multivariable functions $\Omega_{\mu+\psi k}\left(y_{1}, \ldots, y_{r}\right)$, $r \in N$, are expressed as an appropriate product of several simpler functions, the assertions of Theorems 3.1, 3.2, 3.3 and 3.4 can be applied in order to derive various families of multilinear and multilateral generating functions for the multivariable multiple hypergeometric functions ${ }^{(k)} E^{(r)}$ defined by (2.1).

\section{Conflicts of InTEREST}

The author declares that there are no conflicts of interest regarding the publication of this article.

\section{Authors Contributions Statement}

All authors jointly worked on the results and they have read and agreed to the published version of the manuscript. 


\section{REFERENCES}

[1] Abreu, S., Britto, R., Duhr, C., Gardi, E., Matthew, J., From positive geometries to a coaction on hypergeometric functions, Journal of High Energy Physics, 2(2020), 1-45.

[2] Agarwal, R.P., Luo, M.J., Agarwal, P., On the extended Appell Lauricella hypergeometric functions and their applications, Filomat, 31(2017), 3693-3713.

[3] Altin, A., Cekim, B., Sahin, R., On the matrix versions of Appell hypergeometric functions, Quaestiones Mathematicae, 37(2014), 31-38.

[4] Bezrodnykh, S.I., Analytic continuation of Lauricella's functions, $F A(N), F B(N)$ and $F D(N)$, Integral Transforms and Special Functions, 31(2020), 921-940.

[5] Bezrodnykh, S.I., Horn's hypergeometric functions with three variables, Integral Transforms and Special Functions, 32(2021), $207-223$.

[6] Brychkov, Y.A., Saad, N., On some formulas for the Appell function $F 2$ ( $a, b, b$ ';,$\left.c^{\prime} ; w ; z\right)$, Integral Transforms and Special Functions, 25(2014), 111-123.

[7] Brychkov, Y.A., Savischenko, N.V., On some formulas for the Horn functions $H 4\left(a, b ; c, c^{\prime} ; w, z\right)$ and $H 7(c)\left(a ; c, c^{\prime} ; w, z\right)$, Integral Transforms and Special Functions, 32(2021), 1-19.

[8] Choi, J., A generalization of Gottlieb polynomials in several variables, Applied Mathematics Letters, 25(2012), 43-46.

[9] Dwivedi, R., Sahai, V., A note on the Appell matrix functions, Quaestiones Mathematicae, 43(2020), 321-334.

[10] Ergashev, T.G., Fundamental solutions of the generalized Helmholtz equation with several singular coefficients and confluent hypergeometric functions of many variables, Lobachevskii Journal of Mathematics, 41(2020), 15-26.

[11] Erkuş, E., Srivastava, H.M., A unified presentation of some families of multivariable polynomials, Integral Transform. Spec. Funct., 17(2006), $267-273$.

[12] Ernst, T., Some results for q_functions of many variables, Rendiconti del Seminario Matematico della Universitá di Padova, 112(2004), 199235.

[13] Exton, H., Multiple Hypergeometric Functions and Applications, Halsted Press (Ellis Horwood Limited, Chichester), John Wiley and Sons, New York, 1976.

[14] Gürel Yılmaz, Ö., Aktaş, R., Taşdelen, F., On some formulas for the k-analogue of Appell functions and generating relations via k-fractional derivative, Fractal and Fractional, 4(2020), 48.

[15] Hidan, M., Abdalla, M., A note on the Appell hypergeometric matrix function F2, Mathematical Problems in Engineering, (2020).

[16] Horn, J., Hypergeometrische funktionen zweier Veränderlichen, Math. Ann., 105(1931), 381-407.

[17] Jaeger J.C., Hulme H.R., The internal conversion of Gamma rays with the production of electrons and positrons, Proceedings of the Royal Society of London A, 148(1935), 708-728.

[18] Kalla, S.L., Parmar, R.K., Purohit, S.D., Some extensions of Lauricella Functions of sevearal variables, Communications of the Korean Mathematical Society, 30(2015), 239-252.

[19] Korkmaz Duzgun, D., Erkus Duman, E., Extended multivariable fourth type Horn functions, Gazi University Journal of Science, 32(2019), $225-240$.

[20] Korkmaz Duzgun, D., Erkuş Duman, E., Generating functions for the extended multivariable fourth type Horn functions, International Journal of Applied Physics and Mathematics, 10(2020), 65-72.

[21] Lauricella, G., Sulle funzioni ipergeometriche a piu variabili, Rendiconti del Circolo Matematico di Palermo, 7(1983), 111-158.

[22] Liu, S.J., Chyan, C.J., Lu, H.C., Srivastava, H.M., Bilateral generating functions for the Chan-Chyan-Srivastava polynomials and the generalized Lauricella functions, Integral Transforms and Special Functions, 23(2012), 539-549.

[23] Ma, H., Some properties for Appell series F2 over finite fields, Integral Transforms and Special Functions, 30(2019), 992-1003.

[24] Olsson P.O.M., A hypergeometric function of two variables of importance in perturbation theory I and II, Arkiv för Fysik; 30(1965), 187-191, ibid. 29(1965), 459-465.

[25] Ozmen, N., Erkuş Duman, E., Some generating functions for a class of hypergeometric polynomials, Gazi University Journal of Science, 31(2018), 1179-1190.

[26] Özmen, N., Some new properties of generalized Bessel polynomials, Applicationes Mathematicae, 46(2019), 85-98.

[27] Rainville, E.D., Special Functions, The Macmillan Company, New York, 1960.

[28] Scarpello, G.M., Ritelli, D., On computing some special values of multivariate hypergeometric functions, Journal of Mathematical Analysis and Applications, 420(2014), 1693-1718.

[29] Srivastava, H.M., Manocha, H.L., A Treatise on Generating Functions, Halsted Press (Ellis Horwood Limited, Chichester), John Wiley and Sons, New York, 1984.

[30] Upadhyaya, L.M., Remarks on Horn's double hypergeometric functions of matrix arguments, Bulletin of Pure and Applied SciencesMathematics, 1(2011), 11-18. 